\title{
Resummation and Simulation of Soft Gluon Effects beyond Leading Color
}

\author{
Matthew De Angelis (D) \\ Consortium for Fundamental Physics, School of Physics \& Astronomy, University of Manchester, \\ Manchester M13 9PL, United Kingdom \\ Jeffrey R. Forshaw (1) \\ Consortium for Fundamental Physics, School of Physics \& Astronomy, University of Manchester, \\ Manchester M13 9PL, United Kingdom \\ and Erwin Schrödinger Institute for Mathematics and Physics, University of Vienna, 1090 Wien, Austria \\ Simon Plätzer®* \\ Particle Physics, Faculty of Physics, University of Vienna, 1090 Wien, Austria \\ and Erwin Schrödinger Institute for Mathematics and Physics, University of Vienna, \\ 1090 Wien, Austria
}

(Received 30 July 2020; revised 19 October 2020; accepted 15 February 2021; published 17 March 2021)

\begin{abstract}
We present first results of resumming soft gluon effects in a simulation of high-energy collisions beyond the leading-color approximation. We work to all orders in QCD perturbation theory using a new parton branching algorithm. This amplitude evolution algorithm resembles a parton shower that is able to systematically include color-suppressed terms. We find that color-suppressed terms can significantly contribute to jet veto cross sections.
\end{abstract}

DOI: 10.1103/PhysRevLett.126.112001

Introduction.-We present first results from a new Monte Carlo code, CVolver, that simulates high-energy particle collisions [1]. Our analysis here is based on simulated two-jet events and our goal is to improve on the accuracy of existing simulations [2-5] by including color correlations beyond the leading-color approximation. To do so we pursue a new paradigm of evolution at the amplitude level in place of the traditional probabilistic algorithms (see also Refs. [6-11]). In the next section we introduce the theoretical framework, with particular emphasis on our treatment of color. In the following section, we present results for the cross section for the production of a two-jet system with a restriction on the amount of radiation lying in some angular region outside of the jets. This process is sensitive to wide-angle, soft gluon emission and thus provides a good test of the framework.

Summing soft gluon effects.-Particle collisions involving colored particles and a large transfer of momentum $Q$ are computable using perturbative QCD. However, fixedorder perturbation theory is often insufficient due to the presence of large logarithms that compensate the smallness of the perturbative coupling $\alpha_{s}$. In other words, there exist

Published by the American Physical Society under the terms of the Creative Commons Attribution 4.0 International license. Further distribution of this work must maintain attribution to the author(s) and the published article's title, journal citation, and DOI. Funded by SCOAP ${ }^{3}$. terms of order $\alpha_{s}^{n} L^{m}$ where $L$ is some large logarithm and $m \leq 2 n$. Large logarithms can arise if gluons are emitted with a low energy compared to the large momentum transfer, and if the observable is sensitive to those emissions. We refer to these as soft gluon logarithms and, in Ref. [8], we presented an iterative algorithm for summing them to all orders in perturbation theory for general shortdistance scattering processes. In this Letter, we will sum the most important of these logarithms, though the framework is general enough to go beyond this "leading logarithmic approximation." The formalism can be extended to also include logarithms of collinear origin $[9,12]$. The differential cross section for $n$ soft gluon emissions can be written as

$$
d \sigma_{n}=\operatorname{Tr} \mathbf{A}_{n} d \Pi_{n},
$$

where the operators $\mathbf{A}_{n}$ satisfy the recurrence relation,

$$
\mathbf{A}_{n}(E)=\mathbf{V}_{E, E_{n}} \mathbf{D}_{n}^{\mu} \mathbf{A}_{n-1}\left(E_{n}\right) \mathbf{D}_{n \mu}^{\dagger} \mathbf{V}_{E, E_{n}}^{\dagger} \Theta\left(E \leq E_{n}\right),
$$

where $\Theta\left(E \leq E_{n}\right)$ is the Heaviside function. The virtualgluon (Sudakov) operator $\mathbf{V}$ sums over all single-gluon exchanges between pairs of partons to all orders, and has been presented in Refs. $[8,13]$ along with the real emission operator and phase space factor, $\mathbf{D}_{n}$ and $d \Pi_{n}$. The virtual evolution and emission operators contain color charge operators $\mathbf{T}_{j}$, which are in a context-specific representation 
of $\mathrm{SU}(3)_{c}$, depending on the number of hard and soft partons considered at the current state of the algorithm. Soft gluon evolution proceeds iteratively starting from a hard-scattering operator, $\mathbf{H}=|\mathcal{M}\rangle\langle\mathcal{M}|$ with $\mathbf{A}_{0}(E)=$ $\mathbf{V}_{E, Q} \mathbf{H} \mathbf{V}_{E, Q}^{\dagger}$. A general observable $\Sigma$ can be computed using

$$
\Sigma(\mu)=\int \sum_{n} d \sigma_{n} u_{n}\left(k_{1}, k_{2}, \ldots, k_{n}\right),
$$

where the $u_{n}$ are the observable dependent measurement functions and the $k_{i}$ are soft gluon momenta. We suppress the dependence on the hard partons and integration over their phase space. Although we assume energy ordering, this is not essential and the algorithm can readily be adapted to account for a different ordering variable. We should take the limit $\mu \rightarrow 0$ in Eq. (3), though it is also correct to put $\mu=Q_{0}$ if the observable is fully inclusive over gluon emissions with $E<Q_{0}$.

This iterative form of the algorithm is well suited to a Monte Carlo implementation. The kinematic part of the evolution is diagonal and does not pose any new problems. The main challenge is to account for the independent color evolution in the amplitude and the conjugate amplitude. To do this we use the color-flow basis in which quarks and antiquarks are represented by color and anticolor lines (an incoming quark is represented by an anticolor line), while gluons are represented by a pair of color and anticolor lines. A basis vector in the color space is then represented by stating how the color and anticolor lines are connected. Amplitudes are decomposed as

$$
|\mathcal{M}\rangle=\sum_{\sigma} \mathcal{M}_{\sigma}|\sigma\rangle
$$

where $\sigma$ denotes a permutation, which identifies a basis vector $[8,14]$. A general state consisting of $n$ color lines has a basis of dimension $n$ ! corresponding to all possible permutations of the numbers $(1,2, \ldots, n)$. We normalize the basis vectors so that

$$
\langle\alpha \mid \beta\rangle=N_{c}^{n-\#(\alpha, \beta)},
$$

where $\#(\alpha, \beta)$ is the minimum number of pairwise swaps by which the permutations $\alpha$ and $\beta$ differ. This basis is overcomplete and not orthogonal but is very simple to implement and provides excellent opportunities for importance sampling. We introduce a dual basis $\mid \alpha]$ such that $\langle\alpha| \beta]=\left[\alpha|\beta\rangle=\delta_{\alpha \beta}\right.$, where $\delta_{\alpha \beta}$ is unity if the two permutations are equal and zero otherwise. Also, $\sum_{\alpha}|\alpha\rangle[\alpha \mid=\mathbf{1}$. The trace in Eq. (1) is then computed using

$$
\operatorname{Tr} \mathbf{A}_{n}=\sum_{\sigma, \tau}\left[\tau\left|\mathbf{A}_{n}\right| \sigma\right]\langle\sigma \mid \tau\rangle
$$

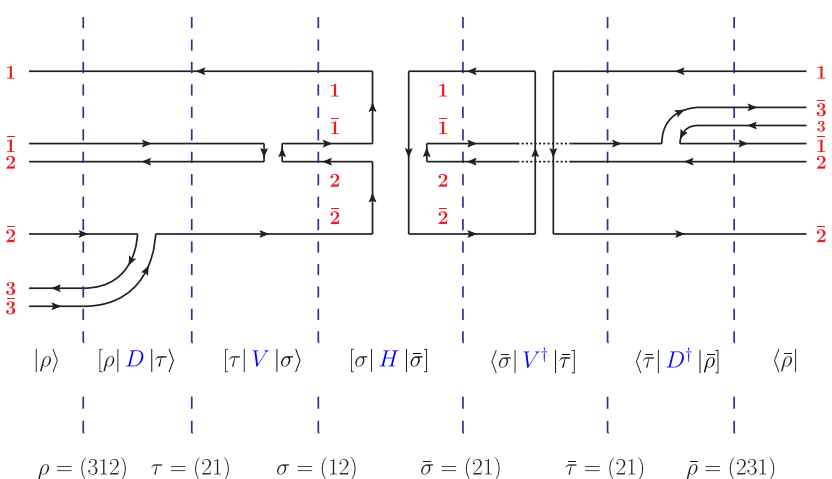

FIG. 1. One contribution to the $\mathbf{A}_{1}$ operator, starting from the hard scattering with two color lines. It corresponds to singlegluon emission with two virtual gluon exchanges. The vertical dotted lines are to help identify the intermediate color states. The algorithm works iteratively outwards, starting from the hard process in the middle and multiplying matrix elements as it goes.

Figure 1 illustrates how we sample over intermediate color states by inserting the unit operator between successive real emission and virtual correction operators. We select initial color flows $\sigma$ and $\bar{\sigma}$ and compute the corresponding hardscattering matrix $[\sigma|\mathbf{H}| \bar{\sigma}]$. As long as the evolution has not terminated, Eq. (2) can be rewritten explicitly in terms of matrix elements such that one step in the evolution is determined by

$$
\begin{aligned}
M_{\rho \bar{\rho}}(E)= & -\frac{\alpha_{s}}{\pi} \frac{d E}{E} \frac{d \Omega}{4 \pi} \sum_{\substack{\tau, \sigma \\
\bar{\tau}, \bar{\sigma}}}\left[\rho\left|\mathbf{D}_{E}\right| \tau\right\rangle\left[\tau\left|\mathbf{V}_{E, E^{\prime}}\right| \sigma\right\rangle \\
& \times M_{\sigma \bar{\sigma}}\left(E^{\prime}\right)\left\langle\bar{\sigma}\left|\mathbf{V}_{E, E^{\prime}}^{\dagger}\right| \bar{\tau}\right]\left\langle\bar{\tau}\left|\mathbf{D}_{E}^{\dagger}\right| \bar{\rho}\right],
\end{aligned}
$$

where $E$ is the energy of the latest emission and $E^{\prime}$ is the previous energy. This expression is the core of our implementation and starting from color flows $(\sigma, \bar{\sigma})$, our Monte Carlo algorithm selects the color flows $(\tau, \bar{\tau})$ after the virtual evolution, the emission scale and momentum, and color flows $(\rho, \bar{\rho})$ after the emission. If the evolution terminates, the final product of matrix elements must be further multiplied by the scalar product matrix $\left\langle\sigma_{m} \mid \bar{\sigma}_{m}\right\rangle$, where $m$ labels the final color flows. This induces a $1 / N_{c}$ suppression factor for every swap by which the final color flows differ.

Calculating the real emission matrix elements and virtual corrections involves computing matrix elements $\left[\tau\left|\mathbf{T}_{i}\right| \sigma\right\rangle$ and $\left[\tau\left|\mathbf{T}_{i} \cdot \mathbf{T}_{j}\right| \sigma\right\rangle$. Explicit expressions for these are presented in Ref. [8]. For the real emissions, the calculations are simplified since the real emission operator can either (a) add a new color line without changing any of the existing color connections or (b) add a new color line and then make a single swap. In the case that the gluon is emitted off a color line, this swap connects the color of the emitted gluon to the anticolor partner of the emitter (and likewise if the gluon is emitted off an anticolor line). Taken 
together, a real emission in the amplitude and the conjugate amplitude only changes the number of swaps by which the two color flows (in the amplitude and conjugate) differ by at most two. Specifically, $0 \leq \#\left(\sigma_{n+1}, \tau_{n+1}\right)-\#\left(\sigma_{n}, \tau_{n}\right) \leq 2$. This means that real emissions never bring the two color flows "closer together." "Singlet" gluons, as emitted in case (a), are subleading in color and inert from the point of view of the subsequent evolution. The simplicity of the real emissions means we do not need to make any approximation when computing them.

The main challenge is to compute the Sudakov matrix elements, $[\tau|\mathbf{V}| \sigma\rangle$, which involves the exponentiation of a possibly large color matrix. The evaluation can be considerably simplified if we are prepared to sum terms accurate only to order $1 / N_{c}^{d}$, where $d$ is a positive integer, while keeping the leading diagonal terms proportional to $\left(\alpha_{s} N_{c}\right)^{n}$ to all orders $n$. Choosing larger values of $d$ will lead to more accurate results that take a longer time to compute. To do this we use the result presented in Ref. [14]:

$$
\begin{aligned}
{[\tau|\mathbf{V}| \sigma\rangle \approx } & \delta_{\tau \sigma} R(\{\sigma\})+\sum_{l=1}^{d}\left(-\frac{1}{N_{c}}\right)^{l} \sum_{\sigma_{0}, \sigma_{1}, \ldots, \sigma_{l}} \delta_{\tau \sigma_{0}} \delta_{\sigma_{l} \sigma} \\
& \times \prod_{\alpha=0}^{l-1} \Sigma_{\sigma_{\alpha} \sigma_{\alpha+1}} R\left(\left\{\sigma_{0}, \sigma_{1}, \ldots, \sigma_{l}\right\}\right) .
\end{aligned}
$$

The anomalous dimension matrix elements are

$$
[\tau|\ln \mathbf{V}| \sigma\rangle=\left(-N_{c} \Gamma_{\sigma}+\frac{\rho}{N_{c}}\right) \delta_{\tau \sigma}+\Sigma_{\tau \sigma} .
$$

Explicit expressions for $\Gamma_{\sigma}, \rho$, and $\Sigma_{\sigma \tau}$ can be computed using the results presented in Refs. $[8,14]$. Note that the offdiagonal contribution $\Sigma_{\tau \sigma}$ is only nonzero if $\#(\sigma, \tau)=1$. In other words, each virtual gluon exchange can either leave a color flow unchanged or induce a single swap. Exponentiating this to produce the Sudakov operator generates the possibility of many swaps, but this can be managed since each swap comes at the price of a factor $1 / N_{c}$. This is what allows us to truncate the sum in Eq. (8) at a small value of $d$. We refer to $d=0$ as our leading-color virtuals $\left(\mathrm{LC}_{V}\right)$ approximation and $d=1$ as next to leading $\left(\mathrm{NLC}_{V}\right)$, etc. The kinematic functions $R$ appearing in Eq. (8) are also listed in Ref. [14] and the $\mathrm{NLC}_{V}$ Sudakov matrix elements are

$$
[\tau|\mathbf{V}| \sigma\rangle=\delta_{\tau \sigma} e^{-N_{c} \Gamma_{\sigma}^{\prime}}-\frac{1}{N_{c}} \Sigma_{\tau \sigma} \frac{e^{-N_{c} \Gamma_{\tau}^{\prime}}-e^{-N_{c} \Gamma_{\sigma}^{\prime}}}{\Gamma_{\tau}^{\prime}-\Gamma_{\sigma}^{\prime}},
$$

with $\Gamma_{\sigma}^{\prime}=\Gamma_{\sigma}-\rho / N_{c}^{2}$, so that keeping only the colordiagonal term generates the leading-color approximation, improved by summing the color-diagonal subleading parts [15].

Note that our $\mathrm{N}^{d} \mathrm{LC}_{V}$ approximation involves at most $d$ swaps for each application of the Sudakov operator, which makes the task of sampling over accessible color states tractable. It should be emphasized that since we treat the real emissions, the scalar product matrix, and the diagonal part of the anomalous dimension matrix without any approximation, our leading-color approximation goes well beyond summing only the strictly leading $(m=0)$ terms in an expansion in $\left(N_{c} \alpha_{s}\right)^{n} / N_{c}^{m}$; see also Ref. [16] for a recent discussion.

One stringent test of the algorithm is that it should correctly handle the cancellation of collinear singular terms. We deal with the collinear region by cutting out small cones around every real emission. Specifically, we impose that $n \cdot n_{i, j}>\lambda$ for emission off the $i j$ pair. Correspondingly, for the loop integrals we regulate using the replacement

$\frac{n_{i} \cdot n_{j}}{n_{i} \cdot n n_{j} \cdot n} \rightarrow \frac{n_{i} \cdot n_{j}}{n \cdot n_{i}+n \cdot n_{j}}\left(\frac{\Theta\left(n \cdot n_{i}-\lambda\right)}{n \cdot n_{i}}+\frac{\Theta\left(n \cdot n_{j}-\lambda\right)}{n \cdot n_{j}}\right)$,

which integrated over solid angle results in $\ln \left(n_{i} \cdot n_{j} / \lambda\right)$, where the only approximation is to disregard terms suppressed by powers of the cutoff $\lambda$. Since the $\ln \lambda$ term is independent of $i$ and $j$, we could exploit color conservation to write

$$
\sum_{i<j}\left(-\mathbf{T}_{i} \cdot \mathbf{T}_{j}\right)=\frac{1}{2} \sum_{i} \mathbf{T}_{i}^{2}
$$

which is color diagonal. This leads to the well-known result that the collinear region has trivial color, and it means that all of the collinear cutoff dependence is in the Abelian sector [9]. For the observable we consider in this Letter, there are only wide-angle, soft logarithms and there is strictly no dependence on the collinear cutoff in the final result.

To perform the evolution efficiently, we use the Sudakov veto algorithm with competition $[17,18]$ in order to select the energy of each emitted gluon. Specifically, for each ordered pair $(i j)$ we compute an energy $E_{i j}$ according to the distribution

$$
d P_{i j}(E)=\frac{d E_{i j}}{E_{i j}} \xi_{i j} \Omega_{i j} \exp \left(-\ln \frac{E}{E_{i j}} \xi_{i j} \Omega_{i j}\right),
$$

where

$$
\Omega_{i j}=\frac{\alpha_{s}}{2 \pi} \ln \frac{n_{i} \cdot n_{j}}{\lambda} .
$$

The energy of the previous emission is $E$ and that of the current emission is $E^{\prime}=\max \left(\left\{E_{i j}\right\}, \mu\right)$. The color flows prior to the emission are $\tau$ and $\bar{\tau}$. The corresponding pair $(i j)$ are the parent partons of the emission, the knowledge of which allows us to select a direction for the emitted 
gluon. The color flows after the emission are selected with probability

$$
P(\rho, \bar{\rho})=\frac{1}{\xi_{i j}} \frac{\langle\rho \mid \bar{\rho}\rangle}{\langle\tau \mid \bar{\tau}\rangle}\left|\left[\rho\left|\mathbf{T}_{i}\right| \tau\right\rangle\left\langle\bar{\tau}\left|\mathbf{T}_{j}\right| \bar{\rho}\right]\right|,
$$

where $\xi_{i j}$ is a normalization.

At present, the algorithm computes the class of observables which restrict angular regions between hard jets by a veto on the energy. We have tailored it to be efficient for this particular observable by terminating the evolution when emitting into the veto region, though this is not a restriction on the general applicability of our approach.

Results.-We consider the production of either a $q \bar{q}$ pair or a pair of gluons $(g g)$, with total energy $2 Q$ in their zero momentum frame. We refer to these as $V \rightarrow q \bar{q}$ (production off a color singlet gauge boson) and $H \rightarrow g g$ (Higgs decay to gluons) though the cross sections we present are independent of the details of the initial state, so long as it is a color singlet. One interesting observable that is sensitive to wide-angle, soft gluon production in events with two primary jets is the "jet veto" cross section, in which one vetoes events that have one or more particles radiated into some fixed angular region with energy greater than some value $Q_{0}$. We will show results using CVolver for events with a veto on particle production in the central region $(-\pi / 4<\theta<\pi / 4)$ in the zero momentum frame of the primary two-jet system. For $q \bar{q}$ production we take $|\mathcal{M}\rangle=|1\rangle$, corresponding to a single color connection, and for $g g$ production we take $|\mathcal{M}\rangle=|21\rangle-|12\rangle / N_{c}$. We denote the corresponding veto cross section $\Sigma(\rho)$, where $\rho=Q_{0} / Q$; i.e., the inclusive cross section is $\Sigma(1)=$ $\operatorname{Tr} \mathbf{H}=N_{c}$ for $q \bar{q}$ production and $\Sigma(1)=N_{c}^{2}-1$ for $g g$ production. The jet veto cross section is an example of a nonglobal observable. These are notoriously difficult to compute beyond the leading-color approximation, though numerical results for the jet veto cross section [19] and the hemisphere jet mass [20] have been obtained using methods very different from those presented here.

Figure 2 shows the veto cross section dependence on $\rho$ for gluon multiplicity $n=2$ in $H \rightarrow g g$. We present results for $d=0\left(\mathrm{LC}_{V}\right)$ and $d=2\left(\mathrm{NNLC}_{V}\right)$. In the case of $g g$ production the differences between $d=1$ and $d=2$ are always less than $1 \%-2 \%$ for up to two emissions or otherwise well within the fluctuations we observe (for three emissions). Also shown are the strictly leading-color results, $\mathrm{LC}_{V+R}$, which are composed of the $N_{c}^{k^{\max }}$ contribution in Eq. (16) below, with $\Sigma_{n}^{\left(k^{\max }\right)}$ calculated in the $d=0$ approximation. Finally, we show the breakdown in terms of the different powers of $N_{c}$ that contribute. Specifically, we consider

$$
\Sigma_{n}=\sum_{k}^{k_{n}^{\max }} N_{c}^{k} \Sigma_{n}^{(k)},
$$

singlet $\rightarrow g g N^{k}$ composition at $n=2$

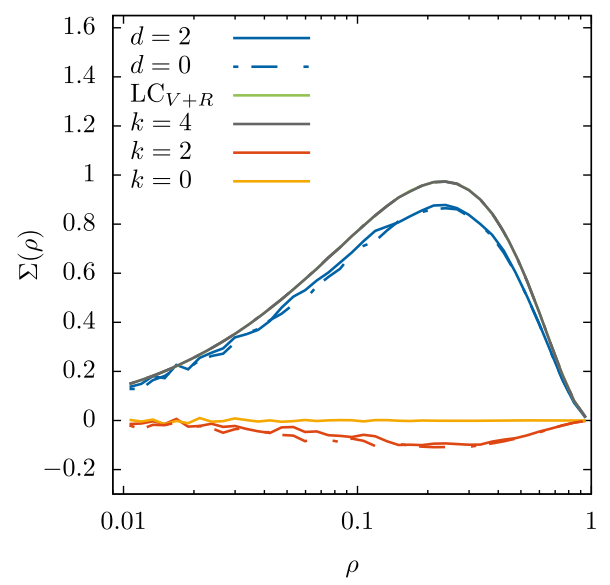

FIG. 2. The jet veto cross section in $H \rightarrow g g$ for two gluon emissions. Also shown are the individual contributions at each order of $N_{c}^{k}$ for $d=0$ and $d=2$. Except for $\mathrm{LC}_{V+R}$, which is in this case equal to the $k=4$ curves, we always compare $d=2$ (solid line) and $d=0$ (dashed line). See text for further details.

where $k_{n}^{\max }=n+1$ for $q \bar{q}$ production and $k_{n}^{\max }=n+2$ for $g g$. The coefficients $\Sigma_{n}^{(k)}$ include perturbatively expanded Sudakov factors, such as Eq. (10). This is not a strict expansion in powers of $N_{c}$. Rather it keeps track of $1 / N_{c}$, off-diagonal suppression that occurs in the hardscattering matrix, the scalar product matrix $(\langle\alpha \mid \beta\rangle)$, the real emission operators, and the successive terms (indexed by $l$ ) in Eq. (8). In the case $n=0$, we observe a peculiar feature of our $\mathrm{LC}_{V}$ approximation for $H \rightarrow g g$ : a nonvanishing cross section at small $\rho$, which occurs because of an unphysical $N_{c}^{0}$ contribution that is present at $d=0$ due

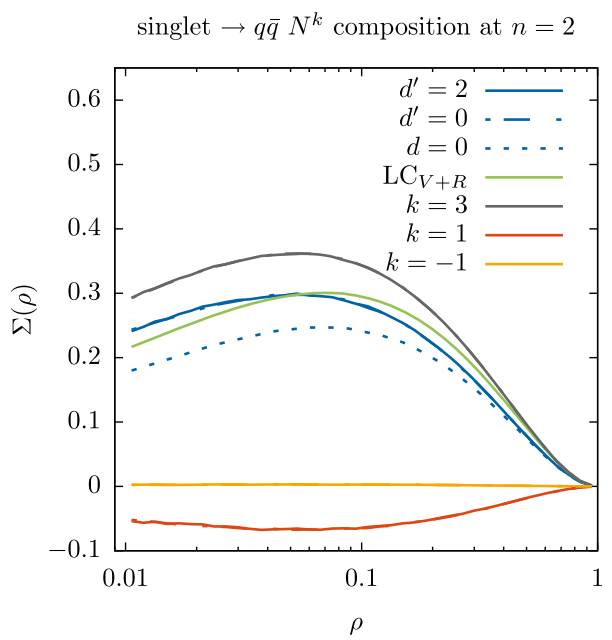

FIG. 3. The jet veto cross section in $V \rightarrow q \bar{q}$ for a gluon multiplicity $n=2$. Also shown are the individual contributions at each order of $N_{c}^{k}$ for $d=0, d^{\prime}=0$, and $d^{\prime}=2$. See text for details. 
singlet $\rightarrow g g$ spectrum

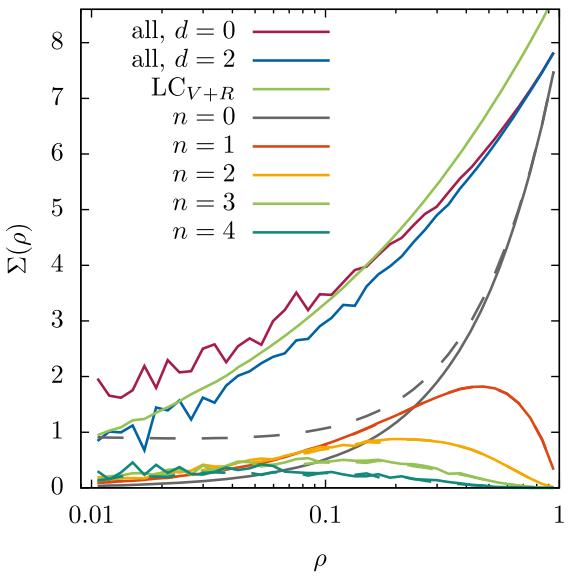

(a)

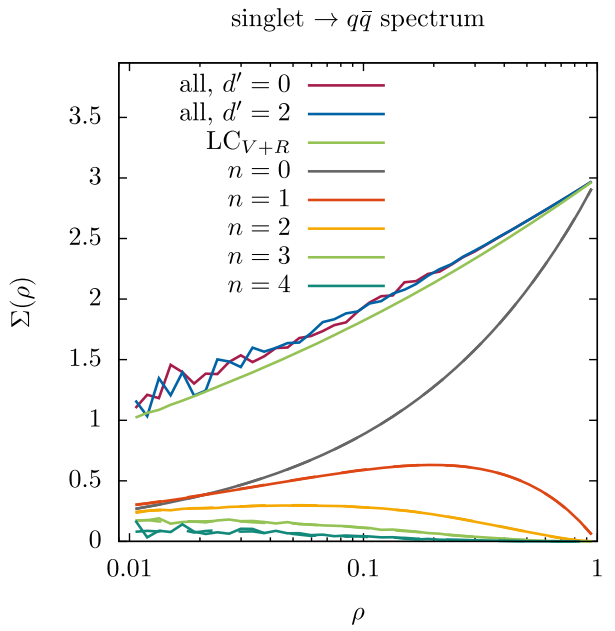

(b)

FIG. 4. The jet veto cross section in (a) $H \rightarrow g g$ and (b) $V \rightarrow q \bar{q}$ for different gluon multiplicities. Results are shown for $d=0$ and $d=2$. We show the contributions from $n=0$ up to 4 emissions; however, the complete result sums over all emissions. (In practice we have limited the simulation to $n \leq 40$ emissions.)

to the subleading $N_{c}$ terms in the scalar product and hardscattering matrices.

Figure 3 is the corresponding plots for the $V \rightarrow q \bar{q}$ process. Here we use the notation $d$ and $d^{\prime}$ to distinguish between exponentiating and not exponentiating the colordiagonal $\rho$ term in the anomalous dimension matrix. We note that for $n=0$, the $d^{\prime}=0$ result is the exact result for quarks and the $n=1, d=0$ result is the exact result for gluons. Finally, in Fig. 4, we show how the total cross section is built up from different multiplicities. The differences between the full-color (solid blue) curves and the leading-color $\left(\mathrm{LC}_{V+R}\right)$ ones are clearly significant, though dominated in the $g g$ case by a factor $8 / 9$ arising from the hard-scattering matrix element. In the $q \bar{q}$ case, the apparent success of our $d^{\prime}=0$ approximation is interesting to note, though we do not expect this to continue once we consider more sophisticated hard processes.

Conclusions.-This Letter represents a major milestone in a project to compute numerically full-color evolution in perturbative QCD. The inclusion of subleading color effects will improve the accuracy of future simulation codes and, as a result, be of considerable value to experimenters and theorists interested in performing percent-level simulations for current and future colliders. For the future, we intend to go beyond the soft approximation and include incoming hadrons.

The authors want to thank the Erwin Schrödinger Institute Vienna for support while this work was being finalized. J. R. F. thanks the Institute for Particle Physics Phenomenology in Durham for the support of an Associateship. This work has received funding from the UK Science and Technology Facilities Council, the European Union's Horizon 2020 research and innovation programme as part of the Marie Skodowska-Curie Innovative Training Network MCnetITN3 (Grant Agreement No. 722104), and in part by the COST actions CA16201 "PARTICLEFACE" and CA16108 "VBSCAN." We are grateful to Thomas Becher, Jack Holguin, Mike Seymour, Malin Sjödahl, and René Ángeles Martínez for discussions.

Note added.-Recently, Ref. [21] presented results that can be directly compared to and agree with those in Fig. 4.

*Corresponding author. simon.plaetzer@univie.ac.at

[1] CVolver stands for color virtual evolver, and originates from the studies first presented in Ref. [14].

[2] M. Bähr et al., Eur. Phys. J. C 58, 639 (2008).

[3] J. Bellm et al., Eur. Phys. J. C 76, 196 (2016).

[4] T. Sjöstrand, S. Ask, J. R. Christiansen, R. Corke, N. Desai, P. Ilten, S. Mrenna, S. Prestel, C. O. Rasmussen, and P.Z. Skands, Comput. Phys. Commun. 191, 159 (2015).

[5] E. Bothmann et al. (Sherpa Collaboration), SciPost Phys. 7, 034 (2019).

[6] Z. Nagy and D. E. Soper, J. High Energy Phys. 06 (2012) 044.

[7] Z. Nagy and D. E. Soper, J. High Energy Phys. 07 (2015) 119.

[8] R. Á. Martínez, M. De Angelis, J. R. Forshaw, S. Plätzer, and M. H. Seymour, J. High Energy Phys. 05 (2018) 044.

[9] J. R. Forshaw, J. Holguin, and S. Plätzer, J. High Energy Phys. 08 (2019) 145.

[10] Z. Nagy and D. E. Soper, Phys. Rev. D 100, 074012 (2019).

[11] Z. Nagy and D. E. Soper, Phys. Rev. D 99, 054009 (2019).

[12] J. R. Forshaw, J. Holguin, and S. Plätzer, J. High Energy Phys. 09 (2020) 014.

[13] We neglect Coulomb-Glauber exchanges, which are needed for processes with two or more colored particles in both the initial and final state of the hard process.

[14] S. Plätzer, Eur. Phys. J. C 74, 2907 (2014). 
[15] The improvement is only relevant if there are quarks in the hard process since $\rho$ always vanishes for purely gluonic states.

[16] J. Holguin, J. R. Forshaw, and S. Plätzer, arXiv:2003.06399.

[17] S. Plätzer and M. Sjodahl, Eur. Phys. J. Plus 127, 26 (2012).
[18] R. Kleiss and R. Verheyen, Eur. Phys. J. C 76, 359 (2016).

[19] Y. Hatta and T. Ueda, Nucl. Phys. B874, 808 (2013).

[20] Y. Hagiwara, Y. Hatta, and T. Ueda, Phys. Lett. B 756, 254 (2016).

[21] Y. Hatta and T. Ueda, Nucl. Phys. B962, 115273 (2021). 